\title{
Zusammenbruch der menschlichen Sozialbeziehungen
}

René Bloch

Korrespondenz:

Dr. med. René Bloch Admiral Parc

75, chemin de l'Argile F-13010 Marseille

marc.girard8[at]wanadoo.fr

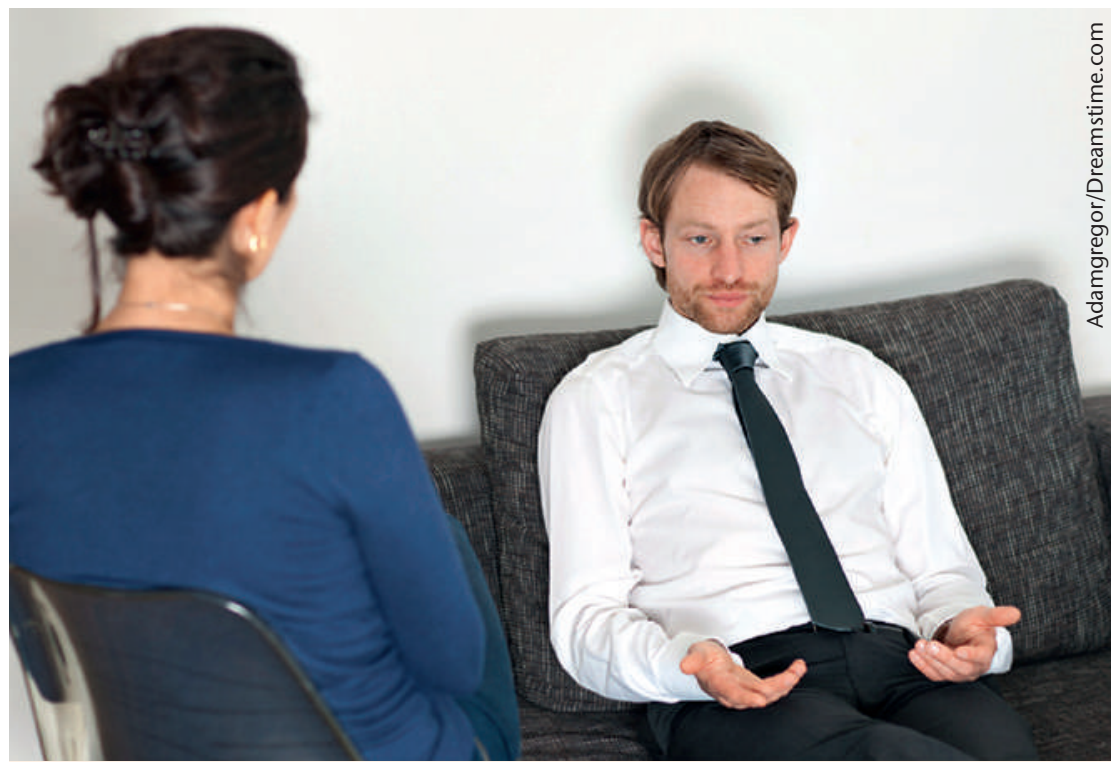

Paradigmenwechsel in der Psychotherapie gefordert: Suchen von Krankheitsursachen im soziokulturellen Umfeld des Patienten, nicht nur in ihm selbst.
Der Mensch ist ein soziales Wesen und bedarf der Sozialbeziehungen, eines Du wie der Luft zum Atmen. Normale Sozialbeziehungen innerhalb der Familie und der Gesellschaft tragen zur Gehirnreifung und Formung des Charakters bei. Wenn die Sozialbeziehungen ungenügend sind und ein regelmässiger Kontakt zu menschlichen Partnern fehlt, kommt es zu einer Dystrophie, d.h. Schmälerung der seelischen Funktionen, und es stellen sich Angstgefühle, Aggressionstendenzen und Süchte ein. Der Schaden für die Seele des Menschen ist umso bedeutender, je früher der Entzug der Beziehungsmöglichkeiten sich einstellt. Dagegen können, wie es gewisse Erfahrungen gezeigt haben, erwachsene Menschen, ohne schwereren und bleibenden Schaden zu erleiden, über Jahre hinweg sequestriert bleiben.

\section{Gesellschaftlicher Wandel}

Mit dem Anbruch des Industriezeitalters im neunzehnten Jahrhundert kam es zu einer weltweiten Migrationsbewegung aus ländlichen Gebieten mit ihren Dörfern in Richtung der Städte. Die Landwirtschaftsproduktion hat sich in den Industrienationen zugunsten einer immer hektischer werdenden industriellen Produktion und technischen Entwicklung verschoben, wobei die städtischen Agglomerationen stark angewachsen sind. Megastädte mit

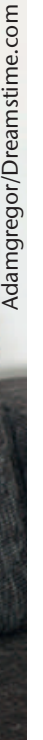

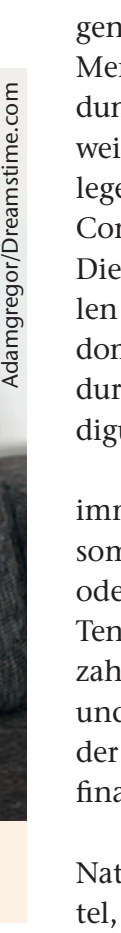


bewirken. Immer häufiger werden deshalb Psychiater sollizitiert, um eine Lösung zu finden. Sie werden von Menschen verschiedener Herkunft um Hilfe angegangen.

\section{Neue Aufgaben für Psychotherapie und Psychiatrie}

Es stellt sich die Frage, ob Psychiatrie und Psychotherapie diesen neuen Aufgaben gewachsen sind, die durch den Wandel der Gesellschaft seit mehreren Jahrzehnten und vor allem seit der Geburt der Psychoanalyse Anfang des neunzehnten Jahrhunderts entstanden sind.

In den letzten Jahrzehnten hat die Psychiatrie grosse Fortschritte gemacht durch die Einführung von Psychopharmaka, durch die sich die sozialen Probleme allerdings auch nicht lösen lassen. Zahlreiche sozial-psychiatrische Institutionen wurden ins Leben gerufen, die nur eine palliative Unterstützung zu geben imstande sind. Am wenigsten ist es noch nicht allgemein durchsetzen können. Einer solchen Konzeption kommt am weitesten die Ethnopsychiatrie entgegen, die aber noch weitgehend unbekannt geblieben ist.

Unter den heutigen Umständen ist für die Psychotherapie ein Paradigmenwechsel notwendig durch eine Verlagerung des Fokus' der therapeutischen Intervention vom Individuum hin zur Gesellschaft. Anstatt nach pathogenen emotionalen Einflüssen in der frühen Kindheit zu suchen oder nach konstitutionellen und angeborenen Schwächen, muss die Umgebung des Patienten in den Mittelpunkt des Blickfeldes gerückt werden. Die gesellschaftliche Umgebung muss ganzheitlich analysiert werden, um das Zusammenspiel der individuellen Reaktionsbereitschaften mit schädlichen Einflüssen aus der Umwelt zu verstehen und um eine Behandlung durch Beratung und Führung anzuwenden. Diese Therapie, obwohl äusserlich verwandt, ist doch verschieden von der sogenannten «stützenden

\section{Vom Individuum wird Anpassung zu erreichen versucht, entweder mit medikamentöser Therapie oder psychotherapeutischen Anpassungs- methoden wie der Verhaltenstherapie.}

der Psychotherapie mit ihren Hunderten verschiedenen Schulen gelungen, eine Anpassung an die neue Stellung des Menschen in der Gesellschaft zu finden. Eines der deutlichsten Beispiele für die Fehlorientierung der Psychiatrie einschliesslich der Psychotherapie ist die Entstehung der Theorie von der Verletzlichkeit des kranken Menschen. Seit mehreren Jahrzehnten hat diese Theorie eine weite Anhängerschaft gefunden, indem sie den Schwerpunkt für die Krankheitsentstehung in den Anlagen des Menschen sucht und weniger in einer lebensund liebesfeindlichen Umwelt. Vom Individuum
Therapie», da sie aus der Überzeugung heraus handelt, den Patienten nicht infolge seiner Schwächen, sondern infolge der Exposition schädlichen Umwelteinflüssen gegenüber unterstützen zu können.

Die Schulung der Psychotherapeuten muss sich an einem solchen Paradigmenwechsel orientieren durch Einführung neuer Disziplinen für die Ausübung des Berufs des Psychotherapeuten. Die Grundlagen der Psychiatrie, der Psychopharmakologie und der Psychopathologie, d. h. der psychischen Krankheitslehre, sind nach wie vor gültig, müssen aber ergänzt werden durch Kenntnisse in Soziologie, Kul-

\section{Für die Psychotherapie ist ein Paradigmenwechsel notwendig: Verlagerung des Fokus vom Individuum zur Gesellschaft hin.}

wird Anpassung zu erreichen versucht, entweder durch eine medikamentöse Therapie oder in Verbindung mit psychotherapeutischen Anpassungsmethoden, wie z. B. der Verhaltenstherapie. Die Versuche, im soziokulturellen Umfeld die Krankheitsursachen ausfindig zu machen und mit dem Patienten einen rationalen Ausweg aus dem ungünstigen Milieu zu finden, sind selten und haben sich bis heute tur- und Religionsgeschichte. Es ist nötig, eine möglichst breite Basis des Wissens zu schaffen, von der aus die Psychotherapeuten operieren können, um eine Einbeziehung des ganzen gesellschaftlichen Umfeldes eines Menschen zu erreichen und denselben aus seiner Isolierung herauszuführen und einen gangbaren Weg in einem schwierigen Umfeld zu finden. 